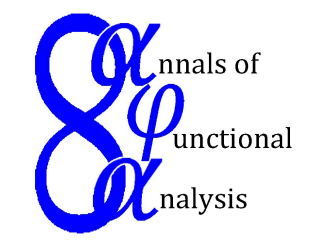

Ann. Funct. Anal. 6 (2015), no. 3, 96-109

http://doi.org/10.15352/afa/06-3-9

ISSN: 2008-8752 (electronic)

http://projecteuclid.org/afa

\title{
ON WEAK EXTERNAL $Q$-HYPERCONVEXITY
}

\author{
OLIVIER OLELA OTAFUDU
}

Communicated by M. A. Japon Pineda

\begin{abstract}
We study a concept of weak external hyperconvexity in quasimetric setting. In this article, we generalize further well-known results about weak external hyperconvexity from metric to quasi-metric point of view. In particular, we show the necessary condition of a subset of $q$-hyperconvex $T_{0^{-}}$ quasi-metric space to be weakly externally $q$-hypeconvex.
\end{abstract}

\section{INTRODUCTION}

In previous work, we started to investigate external hyperconvexity in quasipseudometric spaces, which we called external $q$-hyperconvexity (see [5]). In this article, we study the concept of weak external $q$-hyperconvexity by generalizing some well-known results about weak external hyperconvexity from metric setting to quasi-pseudometric point of view. For instance, we generalize a well-known result of Espínola et al.[1] which states that a subset $D$ of a hyperconvex metric space $(M, d)$ is weakly externally hyperconvex if and only if $D$ is proximinal nonexpansive retract of $D \cup\{z\}$ for any $z \in M \backslash D$.

Our investigations confirm the surprising fact that many classical results about weak external hyperconvexity in metric spaces do not make essential use of the symmetry of the metric and therefore still hold (sometimes in a slightly modified form) for our concept of weak external $q$-hyperconvexity in quasi-pseudometric spaces (see also $[1,3]$ for a more general approach).

Date: Received: Sep. 2, 2014; Revised: Dec. 19, 2014; Accepted: Jan. 9, 2015.

2010 Mathematics Subject Classification. Primary 47H09; Secondary 54E35, 54C15, 54E55.

Key words and phrases. Nonexpansive mapping, $q$-hyperconvexity, proximinal nonexpansive retraction. 


\section{Preliminaries}

This section recalls the most important definitions that we shall use in the following.

Definition 2.1. Let $X$ be a set and let $d: X \times X \rightarrow[0, \infty)$ be a function mapping $X \times X$ into the set $[0, \infty)$ of the nonnegative reals. Then, $d$ is called a quasi-pseudometric on $X$ if

(a) $d(x, x)=0$ whenever $x \in X$,

(b) $d(x, z) \leq d(x, y)+d(y, z)$ whenever $x, y, z \in X$.

We shall say that $d$ is a $T_{0}$-quasi-metric provided that $d$ also satisfies the following condition: For each $x, y \in X$,

$d(x, y)=0=d(y, x)$ implies that $x=y$.

Remark 2.2. Let $d$ be a quasi-pseudometric on a set $X$, then $d^{-1}: X \times X \rightarrow[0, \infty)$ defined by $d^{-1}(x, y)=d(y, x)$ whenever $x, y \in X$ is also a quasi-pseudometric, called the conjugate quasi-pseudometric of $d$. As usual, a quasi-pseudometric $d$ on $X$ such that $d=d^{-1}$ is called a pseudometric. Note that for any $\left(T_{0^{-}}\right)$quasipseudometric $d, d^{s}=\max \left\{d, d^{-1}\right\}=d \vee d^{-1}$ is a pseudometric (metric).

Remark 2.3. We remark that for a quasi-pseudometric space $(X, d)$ :

(1) For each $x \in X$ and $\epsilon>0, B_{d}(x, \epsilon)=\{y \in X: d(x, y)<\epsilon\}$ denotes the open $\epsilon$-ball at $x$.

(2) The collection of all "open" balls yields a base for a topology $\tau(d)$. It is called the topology induced by $d$ on $X$.

(3) Similarly we set for each $x \in X$ and $\epsilon \geq 0, C_{d}(x, \epsilon)=\{y \in X: d(x, y) \leq$ $\epsilon\}$. Note that $C_{d}(x, \epsilon)$ is $\tau\left(d^{-1}\right)$-closed, but not $\tau(d)$-closed in general.

Recall that a $T_{0}$-quasi-metric space $(X, d)$ is said to be bicomplete if the metric space $\left(X, d^{s}\right)$ is complete.

The following definitions can by found in [4] (compare [6]). Note that the weaker version of the concept of $q$-hyperconvexity is the concept of metrical convexity. The notion of metrically convex is defined as follows.

Definition 2.4. Let $(X, d)$ be a quasi-pseudometric space. We say that $X$ is metrically convex if for any points $x, y \in X$ and nonnegative numbers $r$ and $s$ such that $d(x, y) \leq r+s$, there exists $z \in X$ such that $d(x, z) \leq r$ and $d(z, y) \leq s$.

We note that if $(X, d)$ is a metrically convex quasi-pseudometric space, then for any points $x, y \in X$ and nonnegative numbers $r$ and $s$ such that

$$
d(x, y) \leq r+s
$$

we have that

$$
C_{d}(x, r) \cap C_{d^{-1}}(y, s) \neq \emptyset .
$$

Definition 2.5. [4, Definition 2] A quasi-pseudometric space $(X, d)$ is called $q$ hyperconvex (or Isbell-convex) provided that for each family $\left(x_{i}\right)_{i \in I}$ of points in $X$ 
and families $\left(r_{i}\right)_{i \in I}$ and $\left(s_{i}\right)_{i \in I}$ of nonnegative real numbers satisfying $d\left(x_{i}, x_{j}\right) \leq$ $r_{i}+s_{j}$ whenever $i, j \in I$, the following condition holds:

$$
\bigcap_{i \in I}\left(C_{d}\left(x_{i}, r_{i}\right) \cap C_{d^{-1}}\left(x_{i}, s_{i}\right)\right) \neq \emptyset .
$$

Definition 2.6. ([5, Definition 5.1]) Let $(X, d)$ be a quasi-pseudometric space. Given a subset $A$ of $X$, we define for $\epsilon_{1}, \epsilon_{2} \geq 0$ the $\epsilon_{1}, \epsilon_{2}$-parallel set of $A$ as

$$
N_{\epsilon_{1}, \epsilon_{2}}(A)=\bigcup_{a \in A}\left(C_{d}\left(a, \epsilon_{2}\right) \cap C_{d^{-1}}\left(a, \epsilon_{1}\right)\right) .
$$

(Note that for each $\epsilon>0$ in particular $N_{\epsilon, \epsilon}(A)=\bigcup_{a \in A} C_{d^{s}}(a, \epsilon)$.)

Thus $x \in N_{\epsilon_{1}, \epsilon_{2}}(A)$ if and only if there exists $a \in A$ such that $d(a, x) \leq \epsilon_{2}$ and $d^{-1}(a, x) \leq \epsilon_{1}$.

Let $(X, d)$ be a $q$-hyperconvex quasi-pseudometric space and let $A \subseteq X$. We say that $A$ is a $q$-admissible subset of $X$ if and only if for each family $\left(x_{i}\right)_{i \in I}$ of points in $X$ and families $\left(r_{i}\right)_{i \in I}$ and $\left(s_{i}\right)_{i \in I}$ of nonnegative real numbers we have that $\emptyset \neq A=\bigcap_{i \in I}\left(C_{d}\left(x_{i}, r_{i}\right) \cap C_{d^{-1}}\left(x_{i}, s_{i}\right)\right)$.

The following characterization will be needed.

Lemma 2.7. ([5, Lemma 5.2]) Let $(X, d)$ be a q-hyperconvex quasi-pseudometric space and let $A$ be a q-admissible subset of $X$, say $\emptyset \neq A=\bigcap_{i \in I}\left(C_{d}\left(x_{i}, r_{i}\right) \cap\right.$ $\left.C_{d^{-1}}\left(x_{i}, s_{i}\right)\right)$ with $x_{i} \in X$ and $r_{i}, s_{i}$ nonnegative reals whenever $i \in I \neq \emptyset$. Then for each $\epsilon_{1}, \epsilon_{2} \geq 0$,

$$
N_{\epsilon_{1}, \epsilon_{2}}(A)=\bigcap_{i \in I}\left(C_{d}\left(x_{i}, r_{i}+\epsilon_{2}\right) \cap C_{d^{-1}}\left(x_{i}, s_{i}+\epsilon_{1}\right)\right) .
$$

We observe that for any subset $A$ of a $q$-hyperconvex quasi-pseudometric space $(X, d)$, we have

$$
N_{\epsilon_{1}, \epsilon_{2}}\left(N_{\delta_{1}, \delta_{2}}(A)\right)=N_{\epsilon_{1}+\delta_{1}, \epsilon_{2}+\delta_{2}}(A)
$$

\section{EXTERNAL $q$-HYPERCONVEXITY}

Next we recall some results mainly from [5] belonging to the theory of the external $q$-hyperconvex spaces which are important in order to understand the weaker version of external $q$-hyperconvex spaces that we are going to introduce in Section 4.

Definition 3.1. Let $(X, d)$ be a quasi-pseudometric space. A subspace $E$ of $(X, d)$ is said to be externally $q$-hyperconvex (relative to $X$ ) if given any family $\left(x_{i}\right)_{i \in I}$ of points in $X$ and families of nonnegative real numbers $\left(r_{i}\right)_{i \in I}$ and $\left(s_{i}\right)_{i \in I}$ the following condition holds:

If $d\left(x_{i}, x_{j}\right) \leq r_{i}+s_{j}$ whenever $i, j \in I$ and $\operatorname{dist}\left(x_{i}, E\right) \leq r_{i}$ and $\operatorname{dist}\left(E, x_{i}\right) \leq s_{i}$ whenever $i \in I$, then $\bigcap_{i \in I}\left(C_{d}\left(x_{i}, r_{i}\right) \cap C_{d^{-1}}\left(x_{i}, s_{i}\right)\right) \cap E \neq \emptyset$.

Example 3.2. ([5, Example 6.2]) Let $E$ be a nonempty externally $q$-hyperconvex subset in a quasi-pseudometric space $(X, d)$ and let $x$ be any point of $X$. Set $\operatorname{dist}(x, E)=r$ and $\operatorname{dist}(E, x)=s$. Then by applying external $q$-hyperconvexity of 
$E$ to the double ball $\left(C_{d}(x, r) ; C_{d^{-1}}(x, s)\right)$, we conclude that there is $p \in C_{d}(x, r) \cap$ $C_{d^{-1}}(x, s) \cap E$. Thus $d(x, p)=\operatorname{dist}(x, E)$ and $d(p, x)=\operatorname{dist}(E, x)$.

Lemma 3.3. (compare [5, Lemma 6.3]) Let $(X, d)$ be a q-hyperconvex space and let $x \in X$. Furthermore let $\emptyset \neq A=\bigcap_{i \in I}\left(C_{d}\left(x_{i}, r_{i}\right) \cap C_{d^{-1}}\left(x_{i}, s_{i}\right)\right)$ where $\left(x_{i}\right)_{i \in I}$ is a nonempty family of points in $X$ and $\left(r_{i}\right)_{i \in I}$ and $\left(s_{i}\right)_{i \in I}$ are families of nonnegative reals. Then there is $p \in A$ such that $\operatorname{dist}(x, A)=d(x, p)$ and $\operatorname{dist}(A, x)=d(p, x)$.

\section{WEAK EXTERNAL $q$-HYPERCONVEXITY}

In [1], Espínola et al. showed that a subset $D$ of a hyperconvex metric space $(M, d)$ is weakly externally hyperconvex relative to $(M, d)$ if and only if $D$ is a proximinal nonexpansive retract of $D \cup F$ for any finite set $F \subseteq M \backslash D$. In this section, we extend this result in the framework of quasi-pseudometric spaces. Therefore, it seems natural to introduce the concept of weakly externally hyperconvex relative to a quasi-pseudometric space that we call weaky externally q-hyperconvex.

Definition 4.1. Let $(X, d)$ be a quasi-pseudometric space. A subspace $E$ of $(X, d)$ is said to be weakly externally q-hyperconvex (relative to $X$ ) if $E$ is externally $q$-hyperconvex relative to $E \cup\{z\}$ for each $z \in X$. Precisely, given any family $\left(x_{i}\right)_{i \in I}$ of points in $X$ all but at most one of which lies in $E$, and families of nonnegative real numbers $\left(r_{i}\right)_{i \in I}$ and $\left(s_{i}\right)_{i \in I}$ satisfying

$d\left(x_{i}, x_{j}\right) \leq r_{i}+s_{j}$, with $\operatorname{dist}\left(x_{i}, E\right) \leq r_{i}$ and $\operatorname{dist}\left(E, x_{i}\right) \leq s_{i}$ if $x_{i} \notin E$, it follows that $\bigcap_{i \in I}\left(C_{d}\left(x_{i}, r_{i}\right) \cap C_{d^{-1}}\left(x_{i}, s_{i}\right)\right) \cap E \neq \emptyset$.

In Definition 4.1, if $(X, d)$ is a metric space and $r_{i}=s_{i}$ for all $i \in I$, then we have the definition of weakly external hyperconvexity in the metric sense (see definition [1, Definition 1.3].

Example 4.2. Let $X=[0, \infty)$ the set of nonnegative reals equipped with the $T_{0}$-quasi-metric space $u(x, y)=\max \{x-y, 0\}$. Then $D=[0,1] \subseteq X$ is weakly externally $q$-hyperconvex relative to $X$.

Proof. From [4, Corollary 1], it is known that $(X, u)$ is $q$-hyperconvex space. Note that $C_{u}(x, \delta)=[x-\delta, \infty) \cap[0, \infty)$ and $C_{u^{-1}}(x, \delta)=(\infty, x+\delta] \cap[0, \infty)$ whenever $x \in[0, \infty)$ and $\delta \geq 0$.

For any $x \in X$, consider $r_{x}$ and $s_{x}$ be nonnegative real numbers such that $u(x, y) \leq r_{x}+r_{y}$ whenever $x, y \in X$ and $\operatorname{dist}(x, D) \leq r_{x}$ and $\operatorname{dist}(D, x) \leq s_{x}$ whenever $x>1$.

Suppose that $\bigcap_{x \in F}\left(C_{u}\left(x, r_{x}\right) \cap C_{u^{-1}}\left(x, s_{x}\right)\right) \cap D=\emptyset$ for some finite subset $F$ of $X$. We assume that $F$ is nonempty. Then we have that

$$
\max \left\{x-r_{x}: x \in F\right\}>\min \left\{x+r_{x}: x \in F\right\} .
$$

It follows that there are $x, y \in F$ such that $x-r_{x}>y+s_{y}$, that is $C_{u}\left(x, r_{x}\right) \cap$ $C_{u^{-1}}\left(y, s_{x}\right) \cap D=\emptyset$. Moreover $x>y$. Hence $u(x, y)=x-y>r_{x}+s_{y}-\mathrm{a}$ contradiction. Furthermore $\bigcap_{x \in F}\left(C_{u}\left(x, r_{x}\right) \cap C_{u^{-1}}\left(x, s_{x}\right)\right) \cap D \neq \emptyset$ whenever $F$ is a finite nonempty subset of $X$. Since for any $x \in X, C_{u}\left(x, r_{x}\right) \cap C_{u^{-1}}\left(x, s_{x}\right) \cap D$ 
is compact with respect to the topology $\tau\left(u^{s}\right)$ of $\mathbb{R}$ (note that $u^{s}$ is the restriction the usual metric of reals to $X)$. Therefore $\bigcap_{x \in X}\left(C_{u}\left(x, r_{x}\right) \cap C_{u^{-1}}\left(x, s_{x}\right)\right) \cap D \neq \emptyset$. $\square$

Example 4.3. Consider the $q$-hyperconvex $T_{0}$-quasi-metric space $(\mathbb{R}, u)$ in $[4$, Example 1] where $u(x, y)=\max \{x-y, 0\}$ whenever $x, y \in \mathbb{R}$. Then $A=\{2\}$ is not weakly externally $q$-hyperconvex relative to $[0, \infty)$.

Proof. We observe first that $A=C_{u}(2,0) \cap C_{u^{-1}}(2,0)$. One can easily check that $A$ is externally $q$-hyperconvex relative to $X$.

Moreover, for any $x \in \mathbb{R}$ with $x \neq 2$, set $r_{x}=\frac{1}{4}$ and $s_{x}=\frac{3}{4}$. Then $u(x, y) \leq$ $1=\frac{1}{4}+\frac{3}{4}$ and $\operatorname{dist}(x,\{2\}) \leq r_{x}$ and $\operatorname{dist}(\{2\}, x) \leq s_{x}$ whenever $x \in \mathbb{R}$ with $x \neq 2$. But

$$
\bigcap_{x \in \mathbb{R}, x \neq 2}\left(C_{u}\left(x, r_{x}\right) \cap C_{u^{-1}}\left(x, s_{x}\right)\right) \cap A \subseteq C_{u}\left(-\frac{7}{4}, \frac{1}{4}\right) \cap C_{u^{-1}}\left(-\frac{9}{4}, \frac{3}{4}\right) \cap A=\emptyset,
$$

since

$C_{u}\left(-\frac{7}{4}, \frac{1}{4}\right) \cap C_{u^{-1}}\left(-\frac{9}{4}, \frac{3}{4}\right) \cap A=[-2, \infty) \cap\left(-\infty,-\frac{3}{2}\right] \cap A=\left[-2,-\frac{3}{2}\right] \cap\{2\} \neq \emptyset$.

Therefore $A=\{2\}$ is not weakly externally $q$-hyperconvex relative to $\mathbb{R}$.

Definition 4.4. (compare [2, Definition 2.5.]) Let $(X, d)$ be a quasi-pseudometric space and $E \subseteq X$. We say that $E$ is a proximinal nonexpansive retract of $X$ if there exists a nonexpansive retraction $R$ of $X$ onto $E$ for which $d(x, R(x))=$ $\operatorname{dist}(x, E)$ and $d(R(x), x)=\operatorname{dist}(E, x)$ for each $x \in X$. Thus $d(R(x), R(y)) \leq$ $d(x, y)$ whenever $x, y \in X$.

Lemma 4.5. Suppose $D$ is a weakly externally q-hyperconvex subset of a quasipseudometric space $(X, d)$, and let $z \in X \backslash D$. Then there exists a nonexpansive retraction $r$ of $D \cup\{z\}$ onto $D$ for which $d(z, r(z))=\operatorname{dist}(z, D)$ and $d(r(z), z)=$ $\operatorname{dist}(D, z)$.

Proof. For each $x, y \in D, d(x, y) \leq d(x, z)+d(z, y)$ and $\operatorname{dist}(z, D) \leq d(z, y)$ and $\operatorname{dist}(D, z) \leq d(x, z)$. Therefore by the definition of weak external $q$-hyperconvexity of $D$ we have

$$
\begin{gathered}
J=\left(\bigcap_{x \in D} C_{d}(x, d(x, z)) \cap C_{d^{-1}}(x, d(z, x))\right) \cap \\
\left(C_{d}(z, \operatorname{dist}(z, D)) \cap C_{d^{-1}}(z, \operatorname{dist}(D, z))\right) \cap D \neq \emptyset .
\end{gathered}
$$

It suffices to take $r(x)=x$ for $x \in D$ and $r(z)$ to be any point in $J$.

The next result is a consequence of the previous lemma.

Lemma 4.6. Suppose $D$ is weakly externally q-hyperonvex subset of a quasipseudometric space $(X, d)$, and let $F$ be a finite subset of $X \backslash D$. Then there exists a nonexpansive retraction $r$ of $D \cup\{z\}$ onto $D$ for which $d(z, r(z))=\operatorname{dist}(z, D)$ and $d(r(z), z)=\operatorname{dist}(D, z)$ for each $z \in F$.

Proof. Let $F=\left\{x_{1}, \cdots, x_{n}\right\}$ and suppose the points of $F$ are ordered so that

$$
i<j \Longrightarrow \operatorname{dist}\left(x_{i}, D\right) \leq \operatorname{dist}\left(x_{j}, D\right) \quad \text { and } \operatorname{dist}\left(D, x_{i}\right) \leq \operatorname{dist}\left(D, x_{j}\right) .
$$


By Lemma 4.5 there exists a nonexpansive retraction $r$ of $D \cup\left\{x_{1}\right\}$ onto $D$ with $d\left(x_{1}, r\left(x_{1}\right)\right)=\operatorname{dist}\left(x_{1}, D\right)$ and $d\left(r\left(x_{1}\right), x_{1}\right)=\operatorname{dist}\left(D, x_{1}\right)$. Let $\mu=\operatorname{dist}\left(x_{2}, D\right)$ and $\nu=\operatorname{dist}\left(D, x_{2}\right)$ and set

$$
\begin{gathered}
A\left(x_{2}\right)=\left(\bigcap_{x \in D} C_{d}\left(x, d\left(x, x_{2}\right)\right) \cap C_{d^{-1}}\left(x, d\left(x_{2}, x\right)\right)\right) \cap \\
\left(C_{d}\left(r\left(x_{1}\right), d\left(x_{1}, x_{2}\right)\right) \cap C_{d^{-1}}\left(r\left(x_{1}\right), d\left(x_{2}, x_{1}\right)\right)\right) \cap\left(C_{d}\left(x_{2}, \mu\right) \cap C_{d^{-1}}\left(x_{2}, \nu\right)\right) \cap D .
\end{gathered}
$$

On can check that the family

$\left[\left(C_{d}\left(x, d\left(x, x_{2}\right)\right)\right)_{x \in D},\left(C_{d}\left(r\left(x_{1}\right), d\left(x_{1}, x_{2}\right)\right),\left(C_{d}\left(x_{2}, \mu\right)\right) ;\left(C_{d^{-1}}\left(x, d\left(x_{2}, x\right)\right)\right)_{x \in D}\right.\right.$, $\left.\left(C_{d^{-1}}\left(r\left(x_{1}\right), d\left(x_{2}, x_{1}\right)\right)\right),\left(C_{d^{-1}}\left(x_{2}, \nu\right)\right)\right]$ of double balls, only $\left(C_{d}\left(x_{2}, \mu\right)\right)$ and $\left(C_{d^{-1}}\left(x_{2}, \nu\right)\right)$ are not centered in $D$, and for which the conditions of Definition 4.1 are satisfied. Since $A\left(x_{2}\right) \neq \emptyset$, it is possible to choose $r\left(x_{2}\right) \in A\left(x_{2}\right)$ and conclude the lemma holds for $n=2$. The full conclusion follows by finite induction.

From Lemma 2.7, we know that if $(X, d)$ is a $q$-hyperconvex quasi-pseudometric space and $A$ is a subset of $X$ which is $q$-admissible. Then for any $\epsilon_{1}, \epsilon_{2} \geq 0$ we have that

$$
N_{\epsilon_{1}, \epsilon_{2}}(A)=\bigcap_{i \in I}\left(C_{d}\left(x_{i}, r_{i}+\epsilon_{2}\right) \cap C_{d^{-1}}\left(x_{i}, s_{i}+\epsilon_{1}\right)\right) .
$$

Moreover, for any subset $A$ of a $q$-hyperconvex quasi-pseudometric space $(X, d)$, we have

$$
N_{\epsilon_{1}, \epsilon_{2}}\left(N_{\delta_{1}, \delta_{2}}(A)\right)=N_{\epsilon_{1}+\delta_{1}, \epsilon_{2}+\delta_{2}}(A) .
$$

Theorem 4.7. Let $(X, d)$ be a q-hyperconvex quasi-pseudometric space and $D \subseteq$ $X$. Then $D$ is weakly externally q-hyperconvex if and only if $D$ is a proximinal nonexpansive retract of $D \cup F$ for any finite $F \subseteq X \backslash D$.

Proof. The necessary condition follows from Lemma 4.5 and Lemma 4.6.

To prove the converse, it suffices to show that if $D$ is not weakly externally $q$-hyperconvex then there exists $z \in X \backslash D$ such that

$$
\left[C_{d}(z, \operatorname{dist}(z, D)) \cap C_{d^{-1}}(z, \operatorname{dist}(D, z))\right] \cap\left(\bigcap_{x \in X}\left(C_{d}(x, d(x, z)) \cap C_{d^{-1}}(x, d(z, x))\right)\right)=\emptyset .
$$

This clearly implies the non existence of a nonexpansive retraction of $D \cup\{z\}$ onto $D$ which satisfies the condition of Lemma 4.5 . If $D$ is not weakly externally $q$ hyperconvex then by definition there exists $z \in X$, a family $\left(x_{i}\right)_{i \in I}$ of points in $X$ and families of nonnegative real numbers $\left(r_{i}\right)_{i \in I}$ and $\left(s_{i}\right)_{i \in I}$ for which $d\left(x_{i}, x_{j}\right) \leq$ $r_{i}+s_{j}, d\left(x_{i}, z\right) \leq r_{i}+\operatorname{dist}(z, D)$ and $d\left(z, x_{i}\right) \leq s_{i}+\operatorname{dist}(D, z)$, and for which

$$
\left.\left[C_{d}(z, \operatorname{dist}(z, D)) \cap C_{d^{-1}}(z, \operatorname{dist}(D, z))\right] \cap \bigcap_{i \in I}\left(C_{d}\left(x_{i}, r_{i}\right) \cap C_{d^{t}}\left(x_{i}, s_{i}\right)\right)\right] \cap D=\emptyset .
$$

However, since $(X, d)$ is $q$-hyperconvex it must be the case that

$$
\left.D_{1}=\left[C_{d}(z, \operatorname{dist}(z, D)) \cap C_{d^{-1}}(z, \operatorname{dist}(D, z))\right] \cap \bigcap_{i \in I}\left(C_{d}\left(x_{i}, r_{i}\right) \cap C_{d^{t}}\left(x_{i}, s_{i}\right)\right)\right] \neq \emptyset .
$$

If

$$
\left[C_{d}(z, \operatorname{dist}(z, D)) \cap C_{d^{-1}}(z, \operatorname{dist}(D, z))\right] \cap\left(\bigcap_{x \in X}\left(C_{d}(x, d(x, z)) \cap C_{d^{-1}}(x, d(z, x))\right)\right)=\emptyset
$$


we are done. Moreover we proceed as follows. Since $D_{1} \cap D=\emptyset$ we may assume

$$
\operatorname{dist}\left(\left[C_{d}(z, \operatorname{dist}(z, D)) \cap C_{d^{-1}}(z, \operatorname{dist}(D, z))\right] \cap D, D_{1}\right)=d_{1}>0
$$

and

$$
\operatorname{dist}\left(D_{1},\left[C_{d}(z, \operatorname{dist}(z, D)) \cap C_{d^{-1}}(z, \operatorname{dist}(D, z))\right] \cap D\right)=d_{2}>0 .
$$

Then it is possible to choose $w_{1} \in D_{1}$ and

$$
w \in\left[C_{d}(z, \operatorname{dist}(z, D)) \cap C_{d^{-1}}(z, \operatorname{dist}(D, z))\right] \cap D
$$

such that $d\left(w_{1}, w\right)=d_{2}+\epsilon_{2}$ and $d\left(w, w_{1}\right)=d_{1}+\epsilon_{1}$ for sufficiently small $\epsilon_{1}, \epsilon_{2} \geq 0$. By $q$-hyperconvexity of $X$ we have

$$
\begin{gathered}
\mathcal{I}=\left[C_{d}\left(z, \operatorname{dist}(z, D)-\frac{d_{2}+\epsilon_{2}}{2}\right) \cap C_{d^{-1}}\left(z, \operatorname{dist}(D, z)-\frac{d_{1}+\epsilon_{1}}{2}\right)\right] \\
\cap\left[\bigcap_{i \in I}\left(C_{d}\left(x_{i}, r_{i}\right) \cap C_{d^{t}}\left(x_{i}, s_{i}\right)\right)\right] \cap D \neq \emptyset .
\end{gathered}
$$

Let $z_{1} \in \mathcal{I}$ and observe that if $d\left(z_{1}, p\right) \leq \frac{d_{2}+\epsilon_{2}}{2}$, for any $p \in D$ we have that

$$
d(z, p) \leq d\left(z, z_{1}\right)+d\left(z_{1}, p\right)<\operatorname{dist}(z, D)-\frac{d_{2}+\epsilon_{2}}{2}+\frac{d_{2}+\epsilon_{2}}{2}=\operatorname{dist}(z, D)
$$

which is a contradiction. Since $\operatorname{dist}\left(z_{1}, D\right) \leq d\left(z_{1}, w\right) \leq \frac{d_{2}+\epsilon_{2}}{2}$, we conclude $\operatorname{dist}\left(z_{1}, D\right)=\left[C_{d}(z, \operatorname{dist}(z, D)) \cap C_{d^{-1}}(z, \operatorname{dist}(D, z))\right] \cap\left[\bigcap_{i \in I}\left(C_{d}\left(x_{i}, r_{i}\right) \cap C_{d^{t}}\left(x_{i}, s_{i}\right)\right)\right]$ $\cap D$. Similarly if for any $p \in D, d\left(p, z_{1}\right)<\frac{d_{1}+\epsilon_{1}}{2}$ we have

$$
\operatorname{dist}\left(D, z_{1}\right)=\frac{d_{1}+\epsilon_{1}}{2} \text {. }
$$

By the assumption of the theorem, the set

$$
\left[C_{d}(z, \operatorname{dist}(z, D)) \cap C_{d^{-1}}(z, \operatorname{dist}(D, z))\right] \cap\left[\bigcap_{i \in I}\left(C_{d}\left(x_{i}, r_{i}\right) \cap C_{d^{t}}\left(x_{i}, s_{i}\right)\right)\right] \cap D
$$

would have to be nonempty. Furthermore, since $d\left(z_{1}, x_{i}\right) \leq r_{1}+\frac{d_{2}+\epsilon_{2}}{2}$, for each $i \in I$, we have

$$
\begin{gathered}
{\left[C_{d}\left(z_{1}, \operatorname{dist}\left(z_{1}, D\right)\right) \cap C_{d^{-1}}\left(z_{1}, \operatorname{dist}\left(D, z_{1}\right)\right)\right] \cap\left(\bigcap _ { i \in I } \left(C_{d}\left(x_{i}, d\left(x_{1}, z_{1}\right)\right) \cap\right.\right.} \\
\left.\left.C_{d^{-1}}\left(x_{1}, d\left(z_{1}, x_{i}\right)\right)\right)\right) \subseteq\left[C_{d}(z, \operatorname{dist}(z, D)) \cap C_{d^{-1}}(z, \operatorname{dist}(D, z))\right] \\
\left.\cap \bigcap_{i \in I}\left(C_{d}\left(x_{i}, r_{i}+\frac{d_{2}+\epsilon_{2}}{2}\right) \cap C_{d^{-1}}\left(x_{i}, s_{i}+\frac{d_{1}+\epsilon_{1}}{2}\right)\right)\right]
\end{gathered}
$$

and by Lemma 2.7,

$$
\begin{gathered}
{\left[C_{d}(z, \operatorname{dist}(z, D)) \cap C_{d^{-1}}(z, \operatorname{dist}(D, z))\right]} \\
\cap\left[\bigcap_{i \in I}\left(C_{d}\left(x_{i}, r_{i}+\frac{d_{2}+\epsilon_{2}}{2}\right) \cap C_{d^{-1}}\left(x_{i}, s_{i}+\frac{d_{1}+\epsilon_{1}}{2}\right)\right)\right] \subseteq N_{\frac{d_{1}+\epsilon_{1}}{2}, \frac{d_{2}+\epsilon_{2}}{2}}\left(D_{1}\right) .
\end{gathered}
$$

Clearly this neighborhood of $D_{1}$ connot intersect

$$
\left[C_{d}(z, \operatorname{dist}(z, D)) \cap C_{d^{-1}}(z, \operatorname{dist}(D, z))\right] \cap D
$$


for $\epsilon_{1}, \epsilon_{2} \geq 0$ sufficiently small, so we conclude

$$
\begin{gathered}
{\left[C_{d}\left(z_{1}, \operatorname{dist}\left(z_{1}, D\right)\right) \cap C_{d^{-1}}\left(z_{1}, \operatorname{dist}\left(D, z_{1}\right)\right)\right] \cap \bigcap_{i \in I}\left(C_{d}\left(x_{i}, d\left(x_{i}, z_{1}\right) \cap C_{d^{-1}}\left(x_{i}, d\left(z_{1}, x_{i}\right)\right)\right]\right.} \\
\cap D=\emptyset .
\end{gathered}
$$

Corollary 4.8. Let $(X, d)$ be a q-hyperconvex $T_{0}$-quasi-metric space and $D \subseteq$ $X$. Then $D$ is weakly externally q-hyperconvex if and only if $D$ is proximinal nonexpansive retract of $D \cup\{z\}$ for any finite set $\{z\} \subseteq X \backslash D$.

Proof. It follows from Lemma 4.5 and Theorem 4.7.

Example 4.9. Consider the $q$-hyperconvex $T_{0}$-quasi-metric space $([0, \infty), u)$ where $u(x, y)=\max \{x-y, 0\}$. We define a map $R:([0, \infty) \rightarrow([0,1], u)$ by $R(x)=x$ if $x \in[0,1]$ and $R(x)=1$ if $x>1$.

If $x \in[0,1]$, then we have that

$$
u(R(x), x)=u(x, x)=0=\operatorname{dist}([0,1], x)
$$

and

$$
u(x, R(x))=u(x, x)=0=\operatorname{dist}(x,[0,1]) .
$$

If $x>1$, then

$u(R(x), x)=u(1, x)=\max \{1-x, 0\}=0=\inf _{t \in[0,1]} \max \{t-x, 0\}=\operatorname{dist}([0,1], x)$

and

$$
u(x, R(x))=u(x, 1)=x-1=\inf _{t \in[0,1]} \max \{x-t\}=\operatorname{dist}(x,[0,1]) .
$$

Hence, $u(R(x), x)=\operatorname{dist}([0,1], x)$ and $u(x, R(x))=\operatorname{dist}(x,[0,1])$ whenever $x \in$ $[0, \infty)$.

Moreover, we show that $R$ is a nonexpansive map. We have four cases.

Case 1. If $x, y \in[0,1]$, it follows that

$$
u(R(x), R(x))=u(x, y) .
$$

Case 2. If $x \in[0,1]$ and $y>1$, then

$$
u(R(x), R(y))=u(x, 1)=0=u(x, y)
$$

and

$$
u(R(y), R(x))=u(1, x)=1-x \leq y-x=u(y, x) .
$$

Case 3. If $x>1$ and $y \in[0,1]$ is similar to Case 2 .

Case 4. If $x>1$ and $y>1$, then

$$
u(R(x), R(y))=u(1,1)=0 \leq u(x, y) .
$$

Therefore, the interval $[0,1]$ is a proximinal nonexpansive retract of $[0, \infty)$. Furthermore, in light of Theorem 4.7, one can define a proximinal nonexpansive retract from of $[0,1] \cup\{2\}$ to confirm that $[0,1]$ is a weakly externally $q$-hyperconvex relative to $[0, \infty)$ as well as we proved in Example 4.2. 


\section{NONEXPANSIVE RETRACTIONS}

This section deals with externally $q$-hyperconvex subset of a quasi-metrically convex quasi-pseudometric space and nonexpansive retraction maps. We shall prove analogue theorems due to Espínola et al. [1].

Let us first recall the definition of a $T_{0}$-quasi-metric injective space.

Definition 5.1. (compare [4, Definition 8]) Let $\left(Y, d_{Y}\right)$ be a $T_{0}$-quasi-metric space. Then it is called $T_{0}$-quasi-metric injective provided that for any $T_{0}$-quasimetric space $\left(X, d_{X}\right)$, any subspace $\mathrm{A}$ of $\left(X, d_{X}\right)$ and any nonexpansive map $f: A \rightarrow\left(Y, d_{Y}\right), f$ can be extended to a nonexpansive map $g:\left(X, d_{X}\right) \rightarrow\left(Y, d_{Y}\right)$.

It is well-known that any $q$-hyperconvex $T_{0}$-quasi-metric space is $T_{0}$-quasimetric injective (see [4, Theorem 1$]$ ).

Theorem 5.2. (compare [1, Theorem 3.1]) Let $(X, d)$ be a metrically convex $T_{0}$-quasi-metric space. Consider a weakly externally q-hyperconvex subset $A$ of $(X, d)$. Then for each $\epsilon_{1}, \epsilon_{2}>0$ there exists a nonexpansive retraction $R$ from $X$ onto $A$ which has property that if $u \in X \backslash A$ there exists $v \in X \backslash A$ with $d(v, R(v))=$ $\operatorname{dist}(v, A)$ and $d(R(v), v)=\operatorname{dist}(A, v)$ and $d(u, v) \leq \epsilon_{1}$ and $d(v, u) \leq \epsilon_{2}$.

Proof. Let $\epsilon_{1}, \epsilon_{2}>0$ and we set

$$
S_{\epsilon_{1}, \epsilon_{2}}=\left\{u \in X: \operatorname{dist}(u, A)=\epsilon_{2} \quad \text { and } \operatorname{dist}(A, u)=\epsilon_{1}\right\} .
$$

Consider the family

$\mathcal{T}_{\epsilon_{1}, \epsilon_{2}}:\left\{\left(H_{\epsilon_{1}, \epsilon_{2}}, r\right): H_{\epsilon_{1}, \epsilon_{2}} \subseteq S_{\epsilon_{1}, \epsilon_{2}}\right.$ and $r: H_{\epsilon_{1}, \epsilon_{2}} \cup A \rightarrow A$ proximinal nonexpansive retraction $\}$. So $\mathcal{T}_{\epsilon_{1}, \epsilon_{2}} \neq \emptyset$, since from Lemma 4.5, there exists a proximinal nonexpansive retract $r$ with $r: A \cup\{z\} \rightarrow A$. If one partially orders $\mathcal{T}_{\epsilon_{1}, \epsilon_{2}}$ in the usual way $\left(H_{\epsilon_{1}, \epsilon_{2}}, r_{1}\right) \preceq\left(K_{\epsilon_{1}, \epsilon_{2}}, r_{2}\right)$ if and only if $H_{\epsilon_{1}, \epsilon_{2}} \subseteq K_{\epsilon_{1}, \epsilon_{2}}$ and $r_{2}$ is an extension of $\left.r_{1}\right)$, then each chain in $\left(\mathcal{T}_{\epsilon_{1}, \epsilon_{2}}, \preceq\right)$ is bounded above. So by Zorn's Lemma $\left(\mathcal{T}_{\epsilon_{1}, \epsilon_{2}}, \preceq\right)$ has a maximal element which we denote by $\left(H_{\epsilon_{1}, \epsilon_{2}}, r\right)$. We want to show that $H_{\epsilon_{1}, \epsilon_{2}}=S_{\epsilon_{1}, \epsilon_{2}}$. Suppose that there exists $v \in S_{\epsilon_{1}, \epsilon_{2}}$ such that $v \notin H_{\epsilon_{1}, \epsilon_{2}}$, and consider the set

$$
\begin{gathered}
P(v)=\left[\cap_{x \in A}\left(C_{d}(x, d(x, v)) \cap\left(C_{d^{-1}}(x, d(v, x))\right)\right] \cap\right. \\
{\left[\cap_{u \in H_{\epsilon_{1}, \epsilon_{2}}}\left(C_{d}(r(u), d(u, v)) \cap C_{d^{-1}}(r(u), d(v, u))\right)\right] \cap\left[C_{d}\left(v, \epsilon_{2}\right) \cap C_{d^{-1}}\left(v, \epsilon_{1}\right)\right] \cap A .}
\end{gathered}
$$

We first want to show that $P(v) \neq \emptyset$, and in order to do this we need only to show that the family $\left[\left(C_{d}(r(u), d(u, v))_{u \in H_{\epsilon_{1}, \epsilon_{2}}},\left(C_{d}(x, d(x, v))\right)_{x \in A}, C_{d}\left(v, \epsilon_{2}\right)\right.\right.$;

$\left(C_{d^{-1}}(r(u), d(v, u))_{u \in H_{\epsilon_{1}, \epsilon_{2}}},\left(C_{d^{-1}}(x, d(v, x))\right)_{x \in A}, C_{d^{-1}}\left(v, \epsilon_{1}\right)\right]$ of double balls has the mixed binary intersection property.

First note that if $u_{1}, u_{2} \in A$, then

$$
d\left(r\left(u_{1}\right), r\left(u_{2}\right)\right) \leq d\left(u_{1}, u_{2}\right) \leq d\left(u_{1}, v\right)+d\left(v, u_{2}\right) .
$$

Therefore $C_{d}\left(r\left(u_{1}\right), d\left(u_{1}, v\right)\right)$ and $C_{d^{-1}}\left(r\left(u_{2}\right), d\left(v, u_{2}\right)\right)$ intersect by the metric convexity of $(X, d)$.

Furthermore, for each $x, y \in A, d(x, y) \leq d(x, v)+d(v, y)$ then metric convexity of $(X, d)$, we have that $C_{d}(x, d(x, v))$ and $C_{d^{-1}}(x, d(v, x))$ intersect.

Of course, $C_{d}\left(v, \epsilon_{2}\right)$ and $C_{d^{-1}}\left(v, \epsilon_{1}\right)$ intersect. 
Moreover, since $d(u, r(u))=\epsilon_{2}$ and $d(r(u), u)=\epsilon_{1}$. Thus

$$
d(r(u), v) \leq d(r(u), u)+d(u, v) \leq \epsilon_{1}+d(u, v)
$$

and

$$
d(v, r(u)) \leq d(v, u)+d(u, r(u)) \leq d(v, u)+\epsilon_{2} .
$$

Hence, $C_{d}(r(u), d(u, v))$ and $C_{d^{-1}}\left(v, \epsilon_{1}\right)$ intersect by metric convexity of $(X, d)$ as well as $C_{d^{-1}}(r(u), d(v, u))$ and $C_{d}\left(v, \epsilon_{2}\right)$ intersect.

Also, for $x \in A$,

$$
d(r(u), x) \leq d(r(u), r(v))+d(r(v), x) \leq d(u, v)+d(v, x)
$$

and

$$
d(x, r(u)) \leq d(x, r(v))+d(r(v), r(u)) \leq d(x, v)+d(v, u) .
$$

By metric convexity of $(X, d)$, we have that $C_{d}(r(u), d(u, v))$ and $C_{d^{-1}}(x, d(v, x))$ intersect and $C_{d^{-1}}(r(u), d(v, u))$ and $C_{d}(x, d(x, v))$ intersect too.

We have shown that the family $\left[\left(C_{d}(r(u), d(u, v))_{u \in H_{\epsilon_{1}, \epsilon_{2}}},\left(C_{d}(x, d(x, v))\right)_{x \in A}, C_{d}\left(v, \epsilon_{2}\right)\right.\right.$; $\left(C_{d^{-1}}(r(u), d(v, u))_{u \in H_{\epsilon_{1}, \epsilon_{2}}},\left(C_{d^{-1}}(x, d(v, x))\right)_{x \in A}, C_{d^{-1}}\left(v, \epsilon_{1}\right)\right]$ of double balls has the mixed binary intersection property.

Hence $\emptyset \neq P(v) \subseteq A$. Now, let $t \in P(v)$ and define $r^{\prime}: H_{\epsilon_{1}, \epsilon_{2}} \cup\{v\} \rightarrow A$ by setting $r^{\prime}(v)=t$ and $r^{\prime}(z)=r(z)$ if $z \in H_{\epsilon_{1}, \epsilon_{2}} \cup\{v\}$. Then for each $u \in H_{\epsilon_{1}, \epsilon_{2}}$, we have

and

$$
d\left(r^{\prime}(v), r^{\prime}(u)\right)=d(t, r(u)) \leq d(v, u)
$$

$$
d\left(r^{\prime}(u), r^{\prime}(v)\right)=d(r(u), t) \leq d(u, v)
$$

Moreover,

$$
\epsilon_{1}=\inf _{x \in A}(d(x, v)) \leq d\left(r^{\prime}(v), v\right)=d(t, v) \leq \epsilon_{1},
$$

so $\operatorname{dist}(D, v)=\epsilon_{1}$. Similarly, we have $\operatorname{dist}(v, D)=\epsilon_{2}$. Therefore, we conclude that the pair $\left(H_{\epsilon_{1}, \epsilon_{2}} \cup\{v\}, r^{\prime}\right)$ contradicts the maximality of $\left(H_{\epsilon_{1}, \epsilon_{2}}, r\right)$ in $\left(\mathcal{T}_{\epsilon_{1}, \epsilon_{2}}, \preceq\right)$. Consequently, $H_{\epsilon_{1}, \epsilon_{2}}=S_{\epsilon_{1}, \epsilon_{2}}$. Therefore, we conclude that there exists a retraction $r_{\epsilon_{1}, \epsilon_{2}}$ of $S_{\epsilon_{1}, \epsilon_{2}}$ onto $A$ with the property for each $x \in S_{\epsilon_{1}, \epsilon_{2}}, r_{\epsilon_{1}, \epsilon_{2}} \in P(x)$.

We next observe that if $v \in S_{2 \epsilon_{1}, 2 \epsilon_{2}}$, then the set

$$
P^{\prime}(v)=\left[\cap_{x \in A}\left(C_{d}(x, d(x, v)) \cap\left(C_{d^{-1}}(x, d(v, x))\right)\right] \cap\right.
$$

$\left[\cap_{u \in S_{\epsilon_{1}, \epsilon_{2}}}\left(C_{d}(r(u), d(u, v)) \cap C_{d^{-1}}(r(u), d(v, u))\right)\right] \cap\left[C_{d}\left(v, 2 \epsilon_{2}\right) \cap C_{d^{-1}}\left(v, 2 \epsilon_{1}\right)\right] \cap A \neq \emptyset$.

To see that this, for any $u \in S_{2 \epsilon_{1}, 2 \epsilon_{2}}$,

$$
d(v, r(u)) \leq d(v, u)+d(u, r(u))=d(u, v)+\epsilon_{1}<d(v, u)+2 \epsilon_{1}
$$

and

$$
d(r(u), v) \leq d(r(u), u)+d(u, v)=\epsilon_{2}+d(u, v)<d(u, v)+2 \epsilon_{2} .
$$

By selecting a point in $P^{\prime}(v)$ it is possible to extend $r_{\epsilon_{1}, \epsilon_{2}}$ nonexpansively from $S_{\epsilon_{1}, \epsilon_{2}}$ to $S_{\epsilon_{1}, \epsilon_{2}} \cup\{v\}$. The previous argument tells how to extend $r_{\epsilon_{1}, \epsilon_{2}}$ to a nonexpansive retraction $A \cup S_{\epsilon_{1}, \epsilon_{2}} \cup S_{2 \epsilon_{1}, 2 \epsilon_{2}}$ onto $A$ with the property that for each $x \in S_{\epsilon_{1}, \epsilon_{2}} \cup S_{2 \epsilon_{1}, 2 \epsilon_{2}}$,

$$
r_{2 \epsilon_{1}, 2 \epsilon_{2}}(x) \in C_{d}(x, \operatorname{dist}(x, A)) \cap C_{d^{-1}}(x, \operatorname{dist}(A, x)) \cap A .
$$


If we let $S=\cup_{n=1}^{\infty} S_{n \epsilon_{1}, n \epsilon_{2}}$. By using induction and proceeding as above, one sees that there exists a nonexpansive retraction $r$ of $A \cup S$ onto $A$ with the property that for each $x \in S$,

$$
r(x) \in C_{d}(x, \operatorname{dist}(x, A)) \cap C_{d^{-1}}(x, \operatorname{dist}(A, x)) \cap A .
$$

Therefore since $A$ is itself $q$-hyperconvex (injective), we can extend $r$ to a nonexpansive mapping $R: M \rightarrow A$ (by Definition 5.1). The conclusion follows from the fact that if $x \in X \backslash A$ then there exists $u \in S$ such that $d(x, u) \leq \epsilon_{1}$ and $d(u, x) \leq \epsilon_{2}$; thus $d(R(x), R(u)) \leq \epsilon_{1}$ and $d(R(u), R(x)) \leq \epsilon_{2}$.

Remark 5.3. Note that metrical convexity assumption of $(X, d)$ in Theorem 5.2 is very important, otherwise the result can not hold.

The following lemma is needed to prove that $N_{\delta, \epsilon}(A)$ is weakly externally $q$ hyperconvex when $A$ is weakly externally $q$-hyperconvex.

Lemma 5.4. (compare [1, Lemma 3.1]) Let $(X, d)$ be a q-hyperconvex $T_{0}$-quasimetric space and $A \subseteq X$. Suppose that for any $x \in X \backslash A$ and any $\delta \in$ $(0, \operatorname{dist}(x, A))$ and $\epsilon \in(0, \operatorname{dist}(A, x))$ there exists a nonexpansive map

$$
R: A \cup\{x\} \rightarrow N_{\delta, \epsilon}(A) \quad \text { such that } \quad R(u)=u, \text { for all } u \in A
$$

and

$$
R(x) \in\left[C_{d}\left(x, \operatorname{dist}\left(x, N_{\delta, \epsilon}(A)\right)\right) \cap C_{d^{-1}}\left(x, \operatorname{dist}\left(N_{\delta, \epsilon}(A), x\right)\right)\right] \cap N_{\delta, \epsilon}(A) .
$$

Then $A$ is weakly externally q-hyperconvex.

Proof. Consider $x \in X \backslash A$, We need only to show that there exists a nonexpansive retraction $r: A \cup\{x\} \rightarrow A$ such that $d(r(x), x)=\operatorname{dist}(A, x)$ and $d(x, r(x))=\operatorname{dist}(x, A)$, according to Corollary 4.8 .

In order to obtain such retraction, we choose two families of nonnegative real numbers $\left\{\delta_{n}\right\} \subseteq\left(0, \operatorname{dist}\left(x, N_{\delta, \epsilon}(A)\right)\right)$ and $\left\{\epsilon_{n}\right\} \subseteq\left(0, \operatorname{dist}\left(N_{\delta, \epsilon}(A), x\right)\right)$ so that $\left\{\delta_{n}\right\}$ and $\left\{\epsilon_{n}\right\}$ are decreasing sequences for which $\sum_{n=1}^{\infty} \delta_{n}<\infty$ and $\sum_{n=1}^{\infty} \epsilon_{n}<\infty$. From the assumption, there exists a nonexpansive map

$$
R_{1}: A \cup\{x\} \rightarrow N_{\delta_{1}, \epsilon_{1}}(A) \text { such that } R_{1}(u)=u, \quad \text { for all } u \in A
$$

and

$$
R_{1}(x)=\left[C_{d}\left(x, \operatorname{dist}\left(x, N_{\delta_{1}, \epsilon_{1}}(A)\right)\right) \cap C_{d^{-1}}\left(x, \operatorname{dist}\left(N_{\delta_{1}, \epsilon_{1}}(A), x\right)\right)\right] \cap N_{\delta_{1}, \epsilon_{1}}(A) .
$$

By analogue arguments, there exists a nonexpansive map

$$
R_{2}: A \cup\left\{R_{1}(x)\right\} \rightarrow N_{\delta_{2}, \epsilon_{2}}(A) \quad \text { such that } \quad R_{2}(u)=u, \quad \text { for all } u \in A
$$

and

$$
\begin{aligned}
& R_{2}\left(R_{1}(x)\right)=\left[C_{d}\left(R_{1}(x), \operatorname{dist}\left(R_{1}(x), N_{\delta_{2}, \epsilon_{2}}(A)\right)\right) \cap\right. \\
& \left.C_{d^{-1}}\left(R_{1}(x), \operatorname{dist}\left(N_{\delta_{2}, \epsilon_{2}}(A), R_{1}(x)\right)\right)\right] \cap N_{\delta_{2}, \epsilon_{2}}(A) .
\end{aligned}
$$

By setting $\bar{R}_{2}(x)=\left(R_{2} \circ R_{1}\right)(x)$. Hence by induction for each $n \in \mathbb{N}$, we define a nonexpansive map

$$
R_{n}: A \cup\left\{\bar{R}_{n-1}(x)\right\} \rightarrow N_{\delta_{n}, \epsilon_{n}}(A) \text { such that } R_{n}(u)=u, \quad \text { for all } u \in A
$$


and

$$
\begin{gathered}
R_{n}\left(\bar{R}_{n-1}(x)\right) \in\left[C_{d}\left(\bar{R}_{n-1}(x), \operatorname{dist}\left(\bar{R}_{n-1}(x), N_{\delta_{2}, \epsilon_{2}}(A)\right)\right) \cap\right. \\
\left.C_{d^{-1}}\left(\bar{R}_{n-1}(x), \operatorname{dist}\left(N_{\delta_{2}, \epsilon_{2}}(A), \bar{R}_{n-1}(x)\right)\right)\right] \cap N_{\delta_{2}, \epsilon_{2}}(A) .
\end{gathered}
$$

One shows that $\left\{\bar{R}_{n}(x):=R_{n}\left(\bar{R}_{n-1}(x)\right)\right\}$ is a $d^{s}$-Cauchy sequence and, thus $\left\{\bar{R}_{n}(x)\right.$ is convergent. We obtain the desired retraction $r$ by taking $r(x)=$ $\lim _{n \rightarrow \infty} \bar{R}_{n}(x)$ and $r(u)=u$, for all $u \in A$.

Theorem 5.5. Let $(X, d)$ be a q-hyperconvex $T_{0}$-quasi-metric space and $A \subseteq X$. If $A$ is a weakly externally q-hyperconvex, then for any $\epsilon_{1}, \epsilon_{2}>0, N_{\epsilon_{1}, \epsilon_{2}}(A)$ is also weakly externally q-hyperconvex.

proof. Consider $x \in X \backslash N_{\epsilon_{1}, \epsilon_{2}}(A)$ and let

$$
\delta_{1} \in\left(0, \operatorname{dist}\left(x, N_{\epsilon_{1}, \epsilon_{2}}(A)\right)\right)
$$

and

$$
\delta_{2} \in\left(0, \operatorname{dist}\left(N_{\epsilon_{1}, \epsilon_{2}}(A), x\right)\right) .
$$

To show that $N_{\epsilon_{1}, \epsilon_{2}}(A)$ is weakly externally $q$-hyperconvex, it is sufficient to show that there exists a nonexpansive map $R^{\prime}: N_{\epsilon_{1}, \epsilon_{2}}(A) \cup\{x\} \rightarrow N_{\epsilon_{1}, \epsilon_{2}}(A)$ such that

$$
R^{\prime}(y)=y \quad \text { for all } y \in N_{\epsilon_{1}, \epsilon_{2}}(A)
$$

and

$$
\begin{gathered}
R^{\prime}(x) \in\left[C_{d}\left(x, \operatorname{dist}\left(x, N_{\epsilon_{1}+\delta_{1}, \epsilon_{2}+\delta_{2}}(A)\right)\right) \cap C_{d^{-1}}\left(x, \operatorname{dist}\left(N_{\epsilon_{1}+\delta_{1}, \epsilon_{2}+\delta_{2}}(A), x\right)\right)\right] \cap \\
N_{\epsilon_{1}+\delta_{1}, \epsilon_{2}+\delta_{2}}(A) .
\end{gathered}
$$

Since $(X, d)$ is $q$-hyperconvex hence metrically convex and by Theorem 5.2 ,there exists a nonexpansive retraction $R$ from $X$ onto $A$ such that

$$
d(u, R(u))=\operatorname{dist}(u, A) \quad \text { and } \quad d(R(u), u)=\operatorname{dist}(A, u) \quad \text { for all } \quad u \in X .
$$

Furthermore,

$$
d(u, R(u)) \leq \operatorname{dist}(u, A)+\delta_{1} \quad \text { and } \quad d(R(u), u) \leq \operatorname{dist}(A, u)+\delta_{2}
$$

for all $u \in X$. Moreover, the retraction $R$ may be defined so that

$$
d(x, R(x))=\operatorname{dist}(x, A) \quad \text { and } \quad d(R(x), x)=\operatorname{dist}(A, x) .
$$

If, we consider $S$ such that

$$
\begin{gathered}
S=\left[C_{d}\left(R(x), \delta_{2}+\epsilon_{2}\right) \cap C_{d^{-1}}\left(R(x), \delta_{1}+\epsilon_{1}\right)\right] \cap \\
{\left[C_{d}\left(x, \operatorname{dist}(x, A)-\left(\delta_{1}+\epsilon_{1}\right)\right) \cap C_{d^{-1}}\left(x, \operatorname{dist}(A, x)-\left(\delta_{2}+\epsilon_{2}\right)\right)\right] \cap} \\
{\left[\cap_{u \in N_{\epsilon_{1}, \epsilon_{2}}}\left(C_{d}(u, d(u, x)) \cap C_{d^{-1}}(u, d(x, u))\right)\right] .}
\end{gathered}
$$

To show that $S \neq \emptyset$, it suffices to show that the family

$$
\begin{gathered}
{\left[C_{d}\left(R(x), \delta_{2}+\epsilon_{2}\right), C_{d}\left(x, \operatorname{dist}(x, A)-\left(\delta_{2}+\epsilon_{2}\right)\right),\left(C_{d}(u, d(u, x))_{u \in N_{\epsilon_{1}, \epsilon_{2}}} ;\right.\right.} \\
C_{d^{-1}}\left(R(x), \delta_{1}+\epsilon_{1}\right), C_{d^{-1}}\left(x, \operatorname{dist}(A, x)-\left(\delta_{1}+\epsilon_{1}\right)\right),\left(C_{d^{-1}}(u, d(x, u))_{u \in N_{\epsilon_{1}, \epsilon_{2}}}\right]
\end{gathered}
$$

double balls has the mixed binary intersection property.

We first note that, for $x \in X \backslash N_{\epsilon_{1}, \epsilon_{2}}(A)$,

$$
d(R(x), x)=\operatorname{dist}(A, x)=\epsilon_{2}+\delta_{2}+\operatorname{dist}(A, x)-\left(\epsilon_{2}+\delta_{2}\right)
$$


and

$$
d(x, R(x))=\operatorname{dist}(x, A)=\epsilon_{1}+\delta_{1}+\operatorname{dist}(x, A)-\left(\epsilon_{1}+\delta_{1}\right) .
$$

Therefore $C_{d}\left(R(x), \delta_{2}+\epsilon_{2}\right)$ and $C_{d^{-1}}\left(x, \operatorname{dist}(A, x)-\left(\delta_{2}+\epsilon_{2}\right)\right)$ intersect by metric convexity of $(X, d)$ and $C_{d}\left(x, \operatorname{dist}(x, A)-\left(\delta_{1}+\epsilon_{1}\right)\right)$ and $C_{d^{-1}}\left(R(x), \delta_{1}+\epsilon_{1}\right)$ intersect too.

Moreover, suppose $u \in A$. Then $d(R(x), u)=d(R(x), R(u)) \leq d(x, u)$ and $d(u, R(x))=d(R(u), R(x)) \leq d(u, x)$ since $R$ is nonexpansive. On the other hand, if $u \in N_{\epsilon_{1}, \epsilon_{2}}(A) \backslash A$, then

$$
d(R(x), u) \leq d(R(x), R(u))+d(R(u), u) \leq d(x, u)+\epsilon_{2}+\delta_{2}=\epsilon_{2}+\delta_{2}+d(x, u)
$$

and

$$
d(u, R(x)) \leq d(u, R(u))+d(R(u), R(x)) \leq \epsilon_{1}+\delta_{1}+d(u, x) .
$$

Thus $C_{d}\left(R(x), \delta_{2}+\epsilon_{2}\right)$ and $C_{d^{-1}}\left(u, d^{-1}(x, u)\right)$ intersect by metric convexity on $(X, d)$ and $C_{d^{-1}}\left(R(x), \delta_{1}+\epsilon_{1}\right)$ and $C_{d}(u, d(u, x))$ intersect by metric convexity of $(X, d)$.

We observe that $C_{d}\left(R(x), \delta_{2}+\epsilon_{2}\right)$ and $C_{d^{-1}}\left(R(x), \delta_{1}+\epsilon_{1}\right)$ intersect and $C_{d}(u, d(u, x))$ and $C_{d^{-1}}\left(u, d^{-1}(x, u)\right)$ intersect too.

We have shown that the family

$$
\begin{gathered}
{\left[C_{d}\left(R(x), \delta_{2}+\epsilon_{2}\right), C_{d}\left(x, \operatorname{dist}(x, A)-\left(\delta_{2}+\epsilon_{2}\right)\right),\left(C_{d}(u, d(u, x))_{u \in N_{\epsilon_{1}, \epsilon_{2}}} ;\right.\right.} \\
C_{d^{-1}}\left(R(x), \delta_{1}+\epsilon_{1}\right), C_{d^{-1}}\left(x, \operatorname{dist}(A, x)-\left(\delta_{1}+\epsilon_{1}\right)\right),\left(C_{d^{-1}}(u, d(x, u))_{u \in N_{\epsilon_{1}, \epsilon_{2}}}\right]
\end{gathered}
$$

of double balls has the mixed binary intersection property. Therefore $S \neq \emptyset$.

We note that if $y \in S$, then $d(R(x), y) \leq \delta_{2}+\epsilon_{2}$ and $d(y, R(x)) \leq \delta_{1}+\epsilon_{1}$ therefore $y \in N_{\epsilon_{1}+\delta_{1}, \epsilon_{2}+\delta_{2}}(A)=N_{\epsilon_{1}, \epsilon_{2}}\left(N_{\delta_{1}, \delta_{2}}(A)\right)$.

Now, let $t \in S$ and define $R^{\prime}: N_{\epsilon_{1}, \epsilon_{2}}(A) \cup\{x\} \rightarrow N_{\epsilon_{1}, \epsilon_{2}}(A)$ by setting $R^{\prime}(x)=t$ and $R^{\prime}(u)=u$ if $u \in N_{\epsilon_{1}, \epsilon_{2}}(A)$. Then for each $u \in N_{\epsilon_{1}, \epsilon_{2}}(A)$, we have

$$
d\left(R^{\prime}(x), R^{\prime}(u)\right)=d(t, u) \leq d(x, u)
$$

and

$$
d\left(R^{\prime}(u), R^{\prime}(x)\right)=d(u, t) \leq d(u, x),
$$

so $R^{\prime}$ is nonexpansive map such that $R^{\prime}(u)=u$ for all $u \in N_{\epsilon_{1}, \epsilon_{2}}(A)$ and

$$
\begin{gathered}
R^{\prime}(x) \in\left[C_{d}\left(x, \operatorname{dist}\left(x, N_{\epsilon_{1}+\delta_{1}, \epsilon_{2}+\delta_{2}}(A)\right)\right) \cap C_{d^{-1}}\left(x, \operatorname{dist}\left(N_{\epsilon_{1}+\delta_{1}, \epsilon_{2}+\delta_{2}}(A), x\right)\right)\right] \cap \\
N_{\epsilon_{1}+\delta_{1}, \epsilon_{2}+\delta_{2}}(A) .
\end{gathered}
$$

So $N_{\epsilon_{1}, \epsilon_{2}}(A)$ is weakly externally $q$-hyperconvex by Lemma 5.4 .

Acknowledgement. The author would like to thank the South African National Research Foundation for partial financial support. The author would like also to thank the referee for several suggestions that have clearly improved the presentation of this article. 


\section{REFERENCES}

1. R. Espínola, W.A. Kirk and G. López, Nonexpansive retraction in hyperconvex spaces, J. Math. Anal. Appl. 251 (2000), 557-570.

2. R. Espínola, On selections of the metric projection and best proximity pairs in hyperconvex spaces, An. Univ. Mariae Curie-Sklodowska, LIX (2005), 9-17.

3. R. Espínola and M.A. Khamsi, Introduction to hyperconvex spaces. In: Handbook of metric fixed point theory, 391-435, Kluwer Acad. Publ., Dordrecht, 2001.

4. E. Kemajou, H.-P.A. Künzi and O.O. Otafudu, The Isbell-hull of a di-space, Topol. Appl. 159 (2012), 2463-2475.

5. H.-P.A. Künzi and O.O. Otafudu, q-hyperconvexity in quasi-pseudometric spaces and fixed point theorems, J. Funct. Spaces Appl. 2012, Art. ID 765903, 18 pp.

6. S. Salbany, Injective objects and morphisms, in: Categorical topology and its relation to analysis, algebra and combinatorics (Prague, 1988), World Sci. Publ., 394-409, Teaneck. NJ. 1989.

7. R. Sine, Hyperconvexity and nonexpansive multifunctions, Trans. Amer. Math. Soc. 315 (1989), $755-767$.

8. R. sine, Hyperconvexity and approximate fixed points, Nonlinear Anal. 13 (1989), 863-869.

School of Mathematical Sciences, North-West University (Mafikeng Campus), Mmabatho 2735, South Africa.

E-mail address: olivier.olelaotafudu@nwu.ac.za, olmaolela@gmail.com 